\title{
Isolation of 2 new metabolites, JBIR-74 and JBIR-75, from the sponge-derived Aspergillus sp. fS14
}

Motoki Takagi, Kenichiro Motohashi and Kazuo Shin-ya

The Journal of Antibiotics (2010) 63, 735; doi:10.1038/ja.2010.123

Correction to: The Journal of Antibiotics (2010) 63, 393-395; doi:10.1038/ja.2010.58

The authors of the above article noted an error in publication of this paper (AOP and in July issue) in the list of authors. The name of the second author should have read Keiichiro Motohashi. 